\title{
Malaria Transmission, Infection, and Disease following Sustained Indoor Residual Spraying of Insecticide in Tororo, Uganda
}

\author{
Joaniter I. Nankabirwa, ${ }^{1,2 \star}$ Emmanuel Arinaitwe, ${ }^{1,3}$ John Rek, ${ }^{1}$ Maxwell Kilama, ${ }^{1}$ Timothy Kizza, ${ }^{1}$ Sarah G. Staedke, ${ }^{3}$ \\ Phillip J. Rosenthal, ${ }^{4}$ Isabel Rodriguez-Barraquer, ${ }^{4}$ Jessica Briggs, ${ }^{4}$ Bryan Greenhouse, ${ }^{4}$ Teun Bousema, ${ }^{5,6}$ Chris Drakeley, ${ }^{6}$ \\ David S. Roos, ${ }^{7}$ Sheena S. Tomko, ${ }^{7}$ David L. Smith, ${ }^{8}$ Moses R. Kamya, ${ }^{1,2}$ and Grant Dorsey ${ }^{4}$ \\ ${ }^{1}$ Infectious Diseases Research Collaboration, Kampala, Uganda; ${ }^{2}$ Department of Medicine, Makerere University College of Health Sciences, \\ Kampala, Uganda; ${ }^{3}$ Faculty of Infectious and Tropical Diseases, London School of Hygiene and Tropical Medicine, London, United Kingdom; \\ ${ }^{4}$ Department of Medicine, University of California San Francisco, San Francisco, California; ${ }^{5}$ Department of Medical Microbiology, Radboud \\ University Nijmegen Medical Centre, Nijmegen, The Netherlands; ${ }^{6}$ Department of Immunology and Infection, London School of Hygiene and \\ Tropical Medicine, London, United Kingdom; ${ }^{7}$ On Behalf of ClinEpiDB, Department of Biology, University of Pennsylvania, Philadelphia, \\ Pennsylvania; ${ }^{8}$ Institute for Health Metrics \& Evaluation, University of Washington, Seattle, Washington
}

\begin{abstract}
Tororo, a district in Uganda with historically high malaria transmission intensity, has recently scaled up control interventions, including universal long-lasting insecticidal net distribution in 2013 and 2017, and sustained indoor residual spraying (IRS) of insecticide since December 2014. We describe the burden of malaria in Tororo 5 years following the initiation of IRS. We followed a cohort of 531 participants from 80 randomly selected households in Nagongera subcounty, Tororo district, from October 2017 to October 2019. Mosquitoes were collected every 2 weeks using CDC light traps in all rooms where participants slept, symptomatic malaria was identified by passive surveillance, and microscopic and submicroscopic parasitemia were measured every 4 weeks using active surveillance. Over the 2 years of follow-up, 15,780 female anopheline mosquitos were collected, the majority (98.0\%) of which were Anopheles arabiensis. The daily human biting rate was 2.07 , and the annual entomological inoculation rate was 0.43 infective bites/person/year. Only 38 episodes of malaria were diagnosed (incidence 0.04 episodes/person/year), and there were no cases of severe malaria or malarial deaths. The prevalence of microscopic parasitemia was $1.9 \%$, and the combined prevalence of microscopic and submicroscopic parasitemia was $10.4 \%$, each highest in children aged $5-15$ years $(3.3 \%$ and $14.0 \%$, respectively). After 5 years of intensive vector control measures in Tororo, the burden of malaria was reduced to very low transmission levels. However, a significant proportion of the population remained parasitemic, primarily school-aged children with submicroscopic parasitemia, providing a potential reservoir for malaria transmission.
\end{abstract}

\section{INTRODUCTION}

The scale-up of proven malaria control interventions, including indoor residual spraying (IRS) of insecticide, longlasting insecticidal nets (LLINs), and effective case management with artemisinin-based combination therapies (ACTs), has been associated with marked reductions in the global burden of malaria. However, progress has leveled off in recent years; in 2018 alone, there were an estimated 228 million cases of malaria, of which $93 \%$ were from Africa, where more than $99 \%$ of cases are due to Plasmodium falciparum. ${ }^{1}$ Most worrying is that the malaria cases are on the rise across some high-burden countries in Africa. ${ }^{1}$ Given these worrisome trends, in 2018, the WHO and Roll Back Malaria Partnership to End Malaria launched "high burden to high impact," a new approach that calls for using the most effective tools in a more targeted way to prevent disease and save lives in countries hardest hit. ${ }^{1}$

Uganda is emblematic of high-burden African countries, ranking third in the total number of malaria cases in $2018 .{ }^{1}$ The country has scaled up malaria control throughout the country, including the adoption of artemether-lumefantrine (AL) as first-line treatment for malaria in 2004 and distribution of LLINs through mass universal coverage campaigns in 2013-2014 and 2016-2017. Indoor residual spraying was re-initiated in 2006, after a gap of 40 years, and is currently being implemented in 14 of 135 districts. Although the scale-up of control

\footnotetext{
${ }^{*}$ Address correspondence to Joaniter I. Nankabirwa, Infectious Diseases Research Collaboration, Mulago Hospital Complex, Plot 2C Nakasero Hill, P.O. Box 7475 Kampala, Uganda. E-mail: jnankabirwa@yahoo.co.uk
}

interventions in Uganda has been well documented, data on changes in the burden and epidemiology of malaria are limited. The most comprehensive data come from repeated national Malaria Indicator Surveys (MIS) conducted in 2009, 2014-2015, and 2018-2019, which have documented a decline in the prevalence of microscopic parasitemia in children younger than 5 years from $42 \%^{2}$ to $19 \%^{3}$ to $9 \%$ (2018 MIS, National Malaria Control Program, Uganda Ministry of Health, unpublished data), respectively. However, these declines have not been uniform around the country, and they have been greatest in regions where IRS has been implemented.

Since 2011, our group has been conducting comprehensive malaria surveillance in cohorts from Tororo district, Uganda, an area with historically high transmission intensity that has seen a dramatic decline in the burden of malaria since IRS was first implemented in December 2014. Here, we describe longitudinal measures of malaria transmission, infection, and disease with a focus on the fourth and fifth years after IRS was implemented.

\section{MATERIALS AND METHODS}

Study setting and population level control interventions. Cohort studies and entomological surveillance were conducted in Nagongera subcounty, Tororo district, a predominantly rural area in eastern Uganda. Before 2013, malaria control in Tororo was limited to the distribution of LLINs through antenatal care services, promotion of intermittent preventive treatment during pregnancy, and malaria case management with AL. In November 2013, universal distribution of free LLINs was conducted as part of a national 
campaign, and a similar campaign was repeated in May 2017. Indoor residual spraying with carbamate bendiocarb was first initiated in December 2014-January 2015, with additional rounds administered in June 2015-July 2015 and November 2015-December 2015. In June 2016-July 2016, IRS was administered with the organophosphate pirimiphos-methyl (Actellic), with repeated rounds in June 2017-July 2017, June 2018-July 2018, and March 2019-April 2019.

The primary focus of this report is clinical and entomological surveillance conducted from October 2017 through October 2019 (referred to as "PRISM2" study), but to provide context, we also include previously described summary data from clinical and entomological surveillance conducted from October 2011 through September 2017 in the same study area (referred to as "PRISM1" study). ${ }^{4-7}$ In PRISM1, all children aged $0.5-10$ years were enrolled from 100 houses randomly selected from Nagongera subcounty. Study participants were followed up for all of their healthcare needs in a dedicated study clinic open 7 days per week. Routine visits were conducted every 1-3 months and included the collection of blood for the assessment of parasitemia by microscopy and loopmediated isothermal amplification (LAMP). The cohort was dynamic such that all newly eligible children from participating households were enrolled, and participants who reached 11 years of age were excluded from further follow-up. Mosquito collections were done monthly in all cohort households using CDC light traps. Entomological assessments included quantifying the number of female Anopheles, species identification, and the detection of sporozoites using ELISA.

Primary study population. In October 2017, households that participated in the PRISM1 cohort study and randomly selected new households within Nagongera subcounty were screened and enrolled in the PRISM2 study if they met the following criteria: 1) at least two household members younger than 5 years (or younger than 10 years if enrolled in the prior cohort study), 2) no more than seven permanent residents (or nine permanent residents if enrolled in the prior cohort study), 3) no plans for the household to move from Nagongera subcounty in the next 2 years, and 4) willing to participate in screening for clinical and entomological surveillance. All permanent residents (regardless of age) from enrolled households were screened and enrolled in the cohort study if they met the following criteria: 1) the selected household was considered their primary residence, 2) agreement to come to the study clinic for any febrile illness, 3) agreement to avoid antimalarial medications outside the study, and 4) provision of written informed consent. The cohort was dynamic such that over the course of the study, any permanent residents who joined the household were screened for enrollment. Participants were followed up through October 2019 ( 2 years) unless they were prematurely withdrawn if they met any of the following criteria: 1) permanent movement out of Nagongera subcounty, 2) unable to be located for > 120 days, 3) withdrawal of informed consent, or 4) unable to comply with the study schedule and procedures.

Enrollment and follow-up of cohort study participants. At enrollment, a baseline evaluation which included a detailed medical history, focused physical examination, and blood collected by venipuncture for hemoglobin measurement, thick blood smear, and storage for future molecular studies was conducted. A household survey was conducted to collect information on characteristics of the household and LLIN ownership. Following the household survey, all household members were given a LLIN. Cohort study participants were encouraged to come to a dedicated study clinic open 7 days per week for all their medical care. Routine visits were conducted every 4 weeks and included a standardized evaluation and collection of blood by finger prick/heel stick (if $<6$ months of age) or venipuncture (if aged 6 months and older) for thick blood smear, hemoglobin measurement (every 12 weeks), and storage for future molecular studies. Study participants found to have a fever (tympanic temperature $>38.0^{\circ} \mathrm{C}$ ) or a history of fever in the previous 24 hours at the time of any clinic visit had a thick blood smear read immediately. If the thick blood smear was positive by light microscopy, then the patient was diagnosed with malaria and managed according to national guidelines. ${ }^{8}$ Study subjects who missed their scheduled routine visits were visited at home and requested to come to the study clinic as soon as possible.

Entomological surveillance. Mosquito collections were conducted every 2 weeks in all cohort study households. In each room where cohort study participants slept, a miniature CDC light trap (Model 512; John W. Hock Company, Gainesville, FL) was positioned $1 \mathrm{~m}$ above the floor. Traps were set at $7 \mathrm{PM}$ and collected at $7 \mathrm{AM}$ the following morning. Female Anopheles were identified taxonomically to species level based on morphological criteria according to established taxonomic keys. ${ }^{9}$ Identification of members of the Anopheles gambiae complex was performed by $\mathrm{PCR}^{10}$ for up to 30 mosquitoes randomly selected every 2 weeks. Sporozoites were assessed in all female Anopheles mosquitoes stored with desiccant using a standardized ELISA technique. ${ }^{11}$

Cohort study laboratory evaluations. Thick blood smears were stained with $2 \%$ Giemsa for 30 minutes and evaluated for the presence of asexual and sexual (gametocytes) parasites. Parasite densities were calculated by counting the number of asexual parasites per 200 leukocytes (or per 500, if the count was less than 10 parasites per 200 leukocytes), assuming a leukocyte count of $8,000 / \mu \mathrm{L}$. A thick blood smear was considered negative if examination of 100 high-power fields revealed no asexual parasites. For quality control, all slides were read by a second microscopist, and a third reviewer settled any discrepant readings. Quantitative PCR (qPCR) was performed at the time of enrollment and at each routine visit (every 4 weeks). DNA was extracted using Qiagen spin columns, and extraction products were tested for the presence and quantity of $P$. falciparum DNA via a highly sensitive qPCR assay targeting the multi-copy conserved var gene acidic terminal sequence, with a lower limit of detection of 1 parasite/ $\mathrm{mL} .{ }^{12}$ All samples that were positive for asexual parasites by microscopy but negative for $P$. falciparum by qPCR were tested for the presence of non-falciparum species using nested PCR. ${ }^{13}$ Hemoglobin measurements were performed every 12 weeks using a portable spectrophotometer (HemoCue, Angelholm, Sweden).

Data analysis. All data were collected using standardized case record forms and double-entered using Microsoft Access (Microsoft Corporation, Redmond, WA). Analyses were performed using Stata, version 14 (Stata Corporation, College Station, TX). Baseline descriptive statistics included proportions for categorical variables and mean (SD) or median (range) values for continuous variables. Measures of transmission were based on entomological surveillance data. It was not possible to directly measure the daily human biting rate (HBR); 
therefore, this metric was estimated using the total number of female Anopheles mosquitoes captured/number of CDC light trap collections. Of note, prior work from our group in Uganda has shown that estimated HBRs using the gold standard method of human landing catches was strongly correlated with those made using CDC light traps. ${ }^{14}$ The sporozoite rate was calculated as the number of mosquitoes testing positive for sporozoites/the number of mosquitoes tested. The annual entomological inoculation rate (EIR) was estimated using the product of the daily HBR and the sporozoite rate $\times 365$ days/ year. Estimates of monthly rainfall were obtained from the NASA Tropical Rainfall Measuring Mission Project. ${ }^{15}$ Clinical metrics included measures of disease and infection stratified by age-groups. The incidence of malaria was calculated as the number of episodes of malaria confirmed by microscopy/ person years of observation. The prevalence of microscopic parasitemia or gametocytemia was calculated as the number of routine blood smears positive for asexual parasites or sexual parasites, respectively/total number of routine blood smears done. The prevalence of microscopic or submicroscopic parasitemia was calculated as the number of routine samples positive by microscopy or qPCR/total number of routine assessments. The prevalence of anemia was defined as the proportion of routine samples with hemoglobin values below specific cutoffs based on prior MIS that have been conducted in Uganda.

Data accessibility. Data from both cohort studies are available through a novel open-access clinical epidemiology database resource, ClinEpiDB. ${ }^{16}$ Data for the study conducted from October 2011 through September 2017 (referred to as "PRISM1") can be found at https://clinepidb.org/ce/app/ record/dataset/DS_0ad509829e. Data for the study conducted from October 2017 through October 2019 (referred to as "PRISM2") can be found at https://clinepidb.org/ce/app/ record/dataset/DS_51b40fe2e2.

Ethics statement. Ethical approval was obtained from the Makerere University School of Medicine Research and Ethics Committee, the Uganda National Council of Science and Technology, the London School of Hygiene \& Tropical Medicine Ethics Committee, and the University of California, San Francisco Committee on Human Research.

\section{RESULTS}

Summary findings from the PRISM1 cohort study. From October 2011 through November 2014, before the implementation of IRS, there were two annual peaks in rainfall followed by two annual peaks in vector density characteristic of much of Uganda (Figure 1A). Pre-IRS, the daily HBR was 34.3, the annual EIR 238, and, among all female Anopheles captured, $67 \%$ were An. gambiae s.s., $22 \%$ An. arabiensis, $10 \%$ An. funestus, and $<1 \%$ other Anopheles species. In the first year following IRS with carbamate bendiocarb, the daily HBR had reduced to 3.9 and the annual EIR to 12.4. Over the second and third years of IRS when the formulation was switched to the organophosphate pirimiphos-methyl (Actellic), the daily HBR had reduced further to 1.8 and the annual EIR to 0.9 . Despite continuation of the two annual peaks in rainfall, in the second and third years of IRS, there were only single annual peaks in vector density, between May and June, and more than $90 \%$ of all female Anopheles captured were An. arabiensis.
Longitudinal trends in measures of infection and disease among children aged $0.5-10$ years are presented in Figure 1B. Before the implementation of IRS, the incidence of malaria was 2.96 episodes/person/year, the prevalence of microscopic parasitemia $31.8 \%$, and the combined prevalence of microscopic and submicroscopic parasitemia $67.5 \%$. In the first year following IRS, the incidence of malaria reduced to 0.97 episodes/ person/year, the prevalence of microscopic parasitemia to $22.3 \%$, and the combined prevalence of microscopic and submicroscopic parasitemia to $40.2 \%$. Over the second and third years of IRS, the incidence of malaria reduced further to 0.50 episodes/person/year, the prevalence of microscopic parasitemia to $8.7 \%$, and the combined prevalence of microscopic and submicroscopic parasitemia to $15.8 \%$.

Characteristics of the PRISM2 households and cohort study participants. In October 2017, 413 households were screened and 80 were enrolled, including 33 households from the prior cohort and 47 new households (Figure 2). Of 80 enrolled households, $58(72.5 \%)$ used traditional materials for construction (thatch roof, mud walls, and open eaves), 78 $(97.5 \%)$ had at least one LLIN, and $56(70.0 \%)$ had at least one LLIN per two persons (Table 1). All household members were given an LLIN after enrollment. During the initial screening period (October 2017), 466 participants were enrolled, and an additional 65 were enrolled as they joined the households during the 2-year follow-up period.

A total of 278 participants $(52.4 \%)$ were female, and the median age at enrollment was 8.7 years (interquartile range 3.7-26.5), with approximately equal distribution between those aged $<5$ years, $5-15$ years, and $>15$ years (Table 1 ). The proportion of participants with microscopic parasitemia at enrollment was $5.7 \%, 8.3 \%$, and $1.2 \%$ for those aged $<5$ years, $5-15$ years, and $>15$ years, respectively. The combined proportion of participants with microscopic and submicroscopic parasitemia at enrollment was 9.0\%, 23.3\%, and $19.3 \%$ for those aged $<5$ years, $5-15$ years, and $>15$ years, respectively. At enrollment, 21 participants $(4.0 \%)$ had hemoglobin levels $<11 \mathrm{gm} / \mathrm{dL}$ (all aged $<15$ years), and only one participant's $(0.2 \%)$ hemoglobin was less than $8 \mathrm{gm} / \mathrm{dL}$. A total of 469 participants $(82.7 \%)$ were followed through the end of the study period, and the commonest reason for premature withdrawal was movement out the study area (Figure 2). Three participants died: a 60-year-old male because of chronic liver failure, a 48-year-old female because of pulmonary tuberculosis, and a 6-year-old male because of a diarrheal illness.

Longitudinal measures of transmission. Over the 2 years of follow-up, which roughly corresponded to the fourth and fifth years following the initiation of IRS, a total of 15,780 female Anopheles mosquitoes were collected, resulting in a daily HBR of 2.07. Sporozoites were detected in only nine mosquitoes, and the overall annual EIR was 0.43 . Continuing the trend that started after IRS was initiated, $98 \%$ of mosquitoes collected were from the An. gambiae s.l. complex, and almost $99 \%$ of these were identified as $A n$. arabiensis by PCR (Table 2). Longitudinal measures of transmission are presented in Figure 3. Only one annual peak in vector density was observed between May and June. This peak occurred in the last year of follow-up despite the fact that IRS had been administered 3 months earlier (March-April 2019, just before the annual peak), whereas prior rounds of pirimiphos-methyl were administered in June-July. 

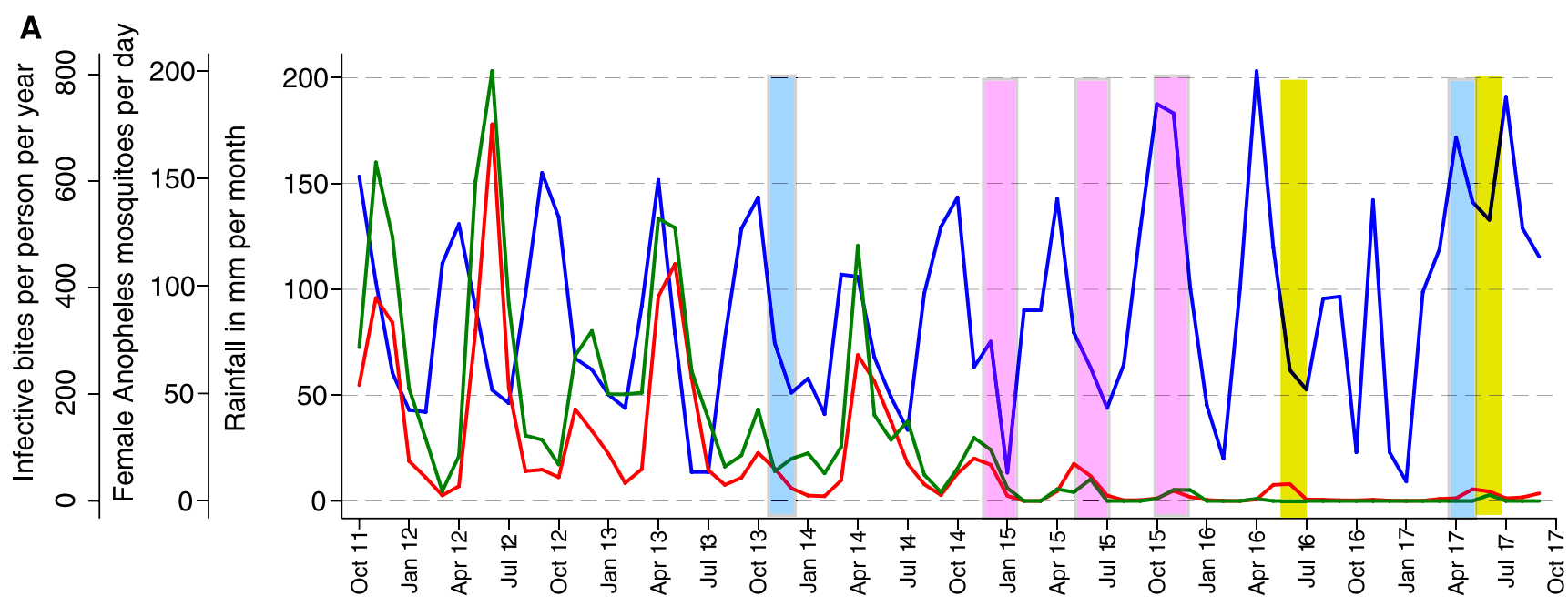

Month and year
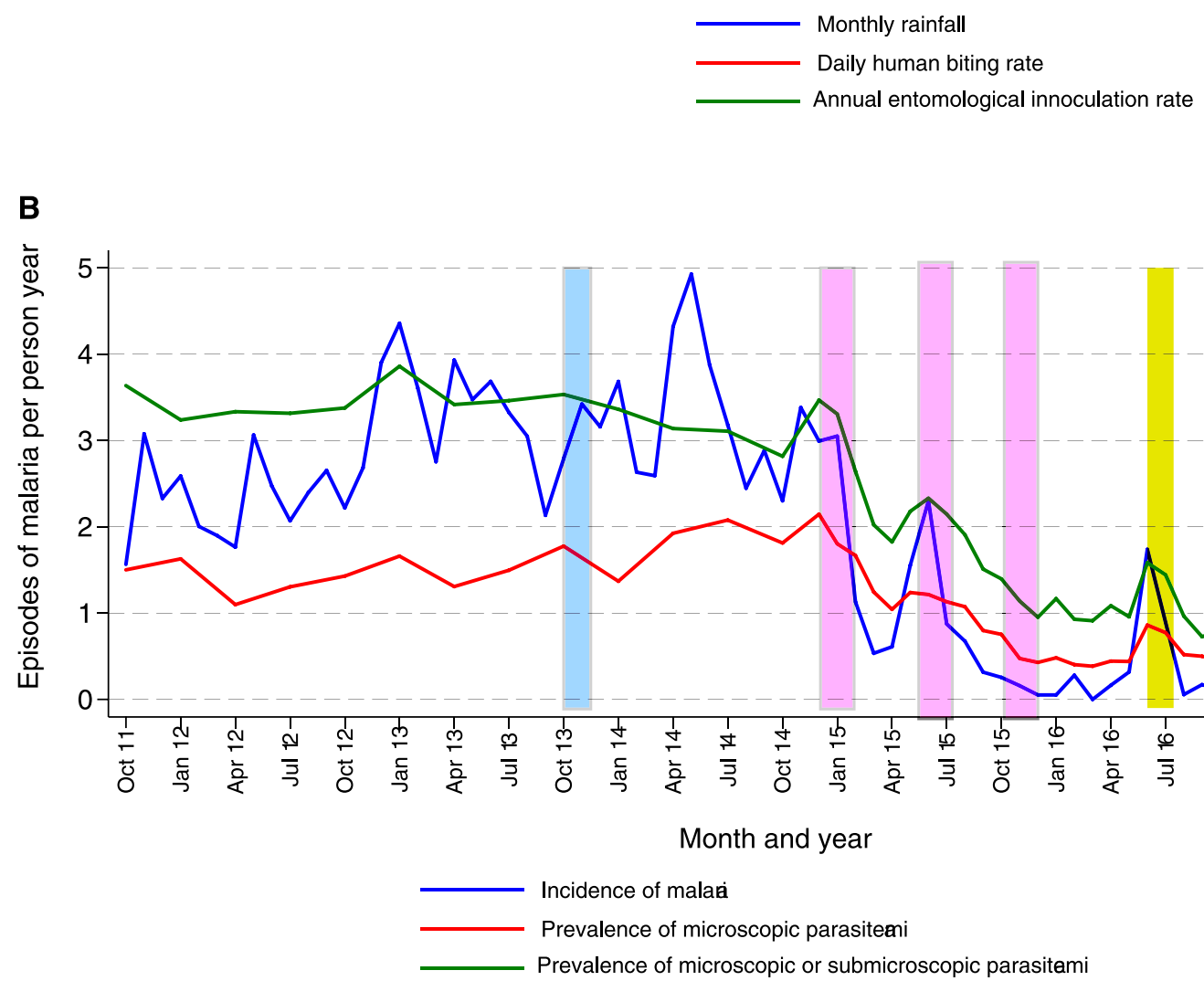

FIGURE 1. Temporal changes in entomological measures of transmission, incidence of malaria, and prevalence of parasitemia from the first cohort study (October 2011-September 2017). (A) Monthly trends of daily human biting rates, annual entomological inoculation rates, and rainfall. (B) Monthly trends of symptomatic malaria, microscopic parasitemia, and combined microscopic and submicroscopic parasitemia. (Blue bars signify rounds of mass long-lasting insecticidal net distribution, pink bars rounds of indoor residual spraying [IRS] with bendiocarb, and yellow bars rounds of IRS with pirimiphos-methyl.) This figure appears in color at www.ajtmh.org.

Longitudinal measures of disease. Only 38 episodes of symptomatic malaria were diagnosed in more than 955 total person years of follow-up, giving an overall incidence of 0.040 episodes/person/year (Table 3). Plasmodium falciparum was detected in 37 cases, and one case was due to Plasmodium malariae. No participants were diagnosed with symptomatic malaria more than once, and no episodes of severe malaria were observed, although two episodes of malaria with danger signs were documented in children younger than 5 years (vomiting and lethargy in a 1-year-old child and lethargy and inability to sit upright in a 3-year-old child). When stratified by age-group, the incidence of malaria was similar in children younger than 5 years (0.048 episodes/person/year) and children aged $5-15$ years ( 0.050 episodes/person/year), but lower among those older than 15 years (0.020 episodes/person/ year). Restricting analyses to children aged $0.5-10$ years, the 


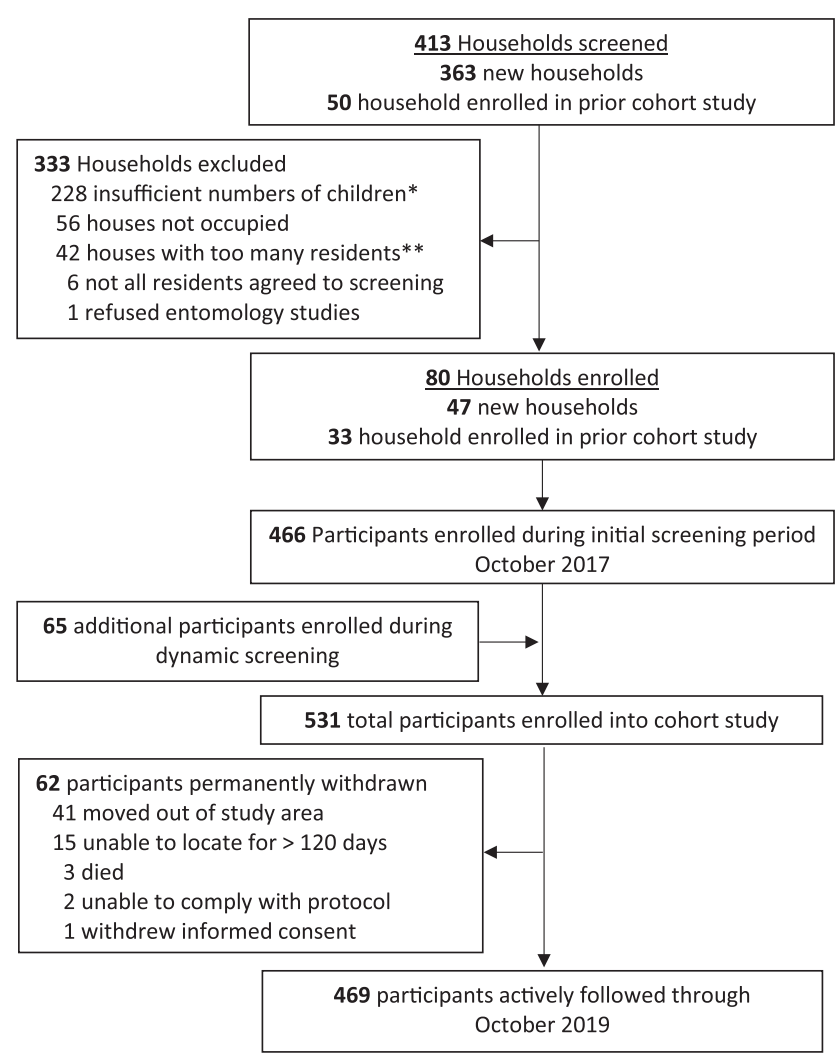

FIGURE 2. Flow diagram of the current cohort study (PRISM2; October 2017-October 2019).

incidence of malaria was 0.054 episodes/person/year compared with 2.96 episodes/person/year before the implementation of IRS from our prior cohort study. Longitudinal trends in malaria cases stratified by age-groups are presented in Figure 4A. A few cases were diagnosed early in the study, and, then, two peaks were seen approximately 1 year apart, roughly correlating with the peaks in vector density, given a 1to 2-month lag. The prevalence of anemia (routine hemoglobin $<11 \mathrm{gm} / \mathrm{dL}$ ) was highest in children younger than 5 years (8.8\%), lower in those aged $5-15$ years (2.0\%), and lowest in those older than 15 years $(0.7 \%)$. The prevalence of moderate to severe anemia (routine hemoglobin $<8 \mathrm{gm} / \mathrm{dL}$ ) was only $0.3 \%$ in children younger than 5 years $(8.8 \%), 0.7 \%$ in those aged $5-15$ years, and $0.1 \%$ in those older than 15 years.

Longitudinal measures of infection. Routine visits were conducted every 4 weeks, resulting in 12,696 total evaluations over the study period. The overall prevalence of microscopic parasitemia was $1.9 \%$, with the highest prevalence in children aged $5-15$ years $(3.3 \%)$, lower in children younger than 5 years $(1.2 \%)$, and lowest in those older than 15 years $(0.8 \%)$ (Table 3). Longitudinal trends in the prevalence of microscopic parasitemia stratified by age-groups are presented in Figure 4B (see https://clinepidb.org/ce/app/workspace/ strategies/import/7eeab4ea9951b3cd for a plot in which you can stratify by other variables). Overall, there was a gradual decrease in the prevalence of microscopic parasitemia during the first 18 months followed by increases during the last 6 months of follow-up. The overall combined prevalence of microscopic and submicroscopic parasitemia was $10.4 \%$, which was more than five times higher than the
TABLE 1

Characteristics of households and cohort participants at enrollment

\begin{tabular}{|c|c|c|}
\hline Characteristic & & Finding \\
\hline \multicolumn{3}{|l|}{ Households $(n=80)$} \\
\hline $\begin{array}{l}\text { Number of household members, } \\
\text { median (range) }\end{array}$ & & $6(3-8)$ \\
\hline Type of housing construction, $n(\%)$ & $\begin{array}{c}\text { Traditional } \\
\text { Modern }\end{array}$ & $\begin{array}{l}58(72.5) \\
22(27.5)\end{array}$ \\
\hline \multicolumn{2}{|l|}{$\begin{array}{l}\text { Number of rooms used for sleeping, } \\
\text { median (range) }\end{array}$} & $2(1-3)$ \\
\hline \multicolumn{2}{|l|}{$\begin{array}{l}\text { Number of sleeping spaces, } \\
\text { median (range) }\end{array}$} & $3(1-7)$ \\
\hline \multicolumn{2}{|l|}{ LLINs per household, mean (SD) } & $3.5(1.6)$ \\
\hline \multicolumn{2}{|l|}{$\begin{array}{l}\text { LLINs per person per household, } \\
\text { mean (SD) }\end{array}$} & $0.61(0.29)$ \\
\hline \multicolumn{2}{|l|}{$\begin{array}{l}\text { Households with at least one LLIN, } \\
n(\%)\end{array}$} & $78(97.5)$ \\
\hline \multicolumn{2}{|l|}{$\begin{array}{l}\text { Households with one LLIN per two } \\
\text { persons, } n(\%)\end{array}$} & $56(70.0)$ \\
\hline \multicolumn{3}{|l|}{ Cohort participants $(n=531)$} \\
\hline \multirow{4}{*}{$\begin{array}{l}\text { Female gender, } n(\%) \\
\text { Age categories (years), } n \text { (\%) }\end{array}$} & & $278(52.4)$ \\
\hline & $<5$ & $177(33.3)$ \\
\hline & $5-15$ & $193(36.4)$ \\
\hline & $>15$ & $161(30.3)$ \\
\hline \multirow{2}{*}{$\begin{array}{l}\text { Microscopic parasitemia stratified by } \\
\text { age categories (years), } n(\%)\end{array}$} & $<5$ & $10(5.7)$ \\
\hline & $\begin{array}{r}5-15 \\
>15\end{array}$ & $\begin{array}{r}16(8.3) \\
2(1.2)\end{array}$ \\
\hline \multirow{3}{*}{$\begin{array}{l}\text { Microscopic or submicroscopic } \\
\text { parasitemia stratified by age } \\
\text { categories (years), } n(\%)\end{array}$} & $<5$ & $16(9.0)$ \\
\hline & $5-15$ & 45 (23.3) \\
\hline & $>15$ & $31(19.3)$ \\
\hline \multirow{3}{*}{$\begin{array}{l}\text { Hemoglobin level stratified by age } \\
\text { categories (years), mean (SD) }\end{array}$} & $<5$ & $12.7(1.3)$ \\
\hline & $5-15$ & $14.2(1.3)$ \\
\hline & $>15$ & $15.9(1.4)$ \\
\hline
\end{tabular}

LLINs = long-lasting insecticidal nets

prevalence of microscopic parasitemia alone. The ratio of submicroscopic to microscopic parasitemia increased with increasing age, which is expected, given the association between age and acquired antimalarial immunity (Table 3). Similar to microscopic parasitemia, the combined prevalence of microscopic and submicroscopic parasitemia was highest among children aged 5-15 years (14.0\%); however, unlike for microscopic parasitemia, prevalence was also relatively high among those older than 15 years $(10.8 \%)$ and lowest in children younger than 5 years (1.9\%). Restricting analyses to children aged $0.5-10$ years, the prevalence of

TABLE 2

Longitudinal entomological metrics using CDC light traps

\begin{tabular}{|c|c|c|c|c|}
\hline \multirow[b]{2}{*}{ Metric } & \multicolumn{4}{|c|}{ Anopheles species group by gross dissection } \\
\hline & All & $\begin{array}{c}\text { An. gambiae } \\
\text { s.l. }\end{array}$ & $\begin{array}{c}\text { An. } \\
\text { funestus }\end{array}$ & $\begin{array}{c}\text { An. } \\
\text { christyi }\end{array}$ \\
\hline $\begin{array}{l}\text { Total number of female } \\
\text { Anopheles collected }\end{array}$ & 15,780 & 15,462 & 38 & 280 \\
\hline Total nights of collections & \multicolumn{4}{|c|}{7,635} \\
\hline Daily human biting rate* & 2.07 & 2.03 & 0.005 & 0.037 \\
\hline Number positive for sporozoites & 9 & 9 & 0 & 0 \\
\hline Sporozoite rate, \% & 0.0006 & 0.0006 & 0 & 0 \\
\hline $\begin{array}{l}\text { Annual entomological } \\
\text { inoculation rate } †\end{array}$ & 0.43 & 0.43 & 0 & 0 \\
\hline $\begin{array}{l}\text { Number tested by PCR for } \\
\text { species }\end{array}$ & N/A & 1,325 & N/A & N/A \\
\hline An. arabiensis, $n(\%)$ & & $\begin{array}{l}1,310 \\
(98.9)\end{array}$ & & \\
\hline An. gambiae s.s., $n(\%)$ & & $15(1.1)$ & & \\
\hline
\end{tabular}




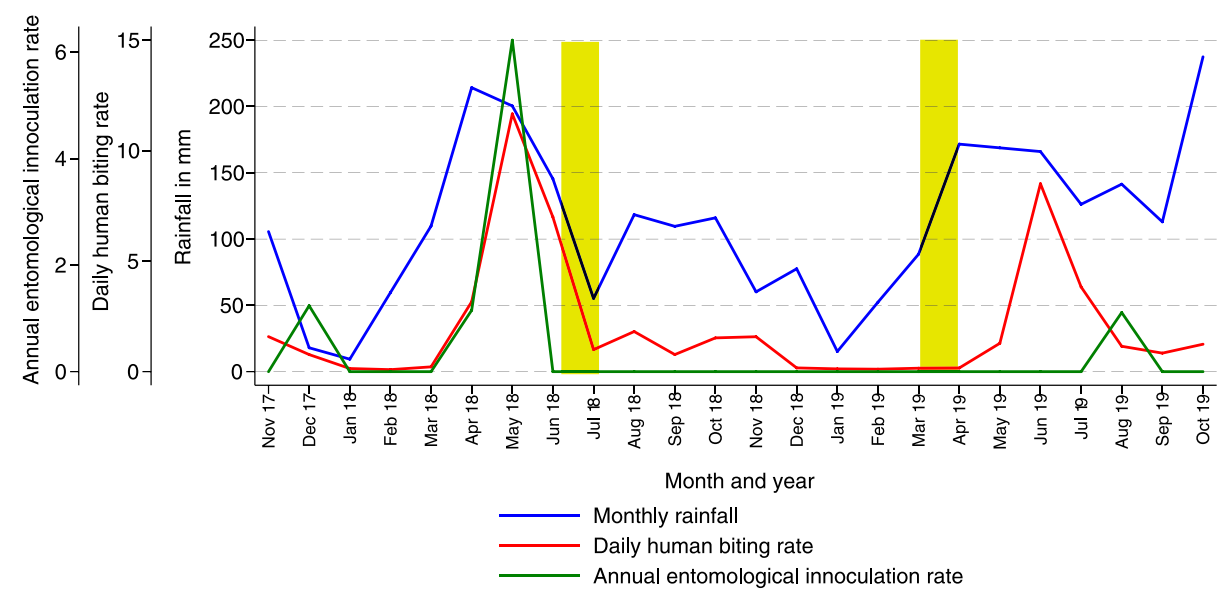

FIGURE 3. Temporal changes in entomological measures of transmission in the current cohort study (October 2017-October 2019). Monthly trends of daily human biting rates, annual entomological inoculation rates, and rainfall in the current study. (Yellow bars signify rounds of indoor residual spraying with pirimiphos-methyl.) This figure appears in color at www.ajtmh.org.

combined microscopic and submicroscopic parasitemia was $6.8 \%$ compared with $67.5 \%$ before the implementation of IRS from our prior cohort study. Longitudinal trends in the prevalence of combined microscopic and submicroscopic parasitemia stratified by age-groups are presented in Figure 4C. Trends were similar to those for microscopic parasitemia, with a gradual decrease in the prevalence of combined microscopic and submicroscopic parasitemia during the first 18 months followed by increases during the last 6 months of follow-up. Interestingly, between May and June 2019, there were no cases of malaria or parasitemia detected by microscopy or qPCR in children younger than 5 years. This 4-month period was followed by an increase in the prevalence of parasitemia, with six cases diagnosed in this age-group.

\section{DISCUSSION}

We describe the epidemiology of malaria following two rounds of universal LLIN distribution and sustained IRS in Tororo, Uganda, a district with historically intense malaria transmission. We studied dynamic cohorts in which all participants received LLINs and prompt treatment of malaria episodes with an ACT. Comparing the period $4-5$ years after IRS initiation with the 3-year period before implementation, we observed 1) a reduction in transmission of more than 500 -fold (annual EIR 0.43 versus 238), 2) a reduction in disease of more than 50-fold (malaria incidence among children aged 0.5-10 years 0.054 versus 2.96), and 3 ) a reduction in infection of $\sim 10$ fold (prevalence of combined microscopic and submicroscopic parasitemia among children aged $0.5-10$ years $6.8 \%$ versus $67.5 \%)$. However, despite the reduction in malaria burden to very low transmission levels, a significant proportion of the population remained parasitemic, providing a potential reservoir for malaria transmission.

The observed decline in transmission intensity was due to a combination of marked reductions in both vector density and the sporozoite rate. When mosquitoes absorb lethal doses of insecticides, their life span is reduced, resulting in declines in vector density and vectoral capacity. ${ }^{17}$ Although there was some evidence of modest declines in transmission intensity following the first round of universal LLIN distribution, marked declines were not seen until after IRS was implemented. We cannot rule out a role for changes in climate in the decreasing malaria burden, ${ }^{18}$ but we did not observe appreciable changes in monthly rainfall over the observation period. Indeed, one

TABLE 3

Longitudinal clinical metrics among cohort participants

\begin{tabular}{|c|c|c|c|c|}
\hline \multirow[b]{2}{*}{ Measure } & \multicolumn{4}{|c|}{ Age-group (years) } \\
\hline & All & $<5$ & $5-15$ & $>15$ \\
\hline Person years of follow-up & 954.5 & 250.4 & 400.0 & 304.4 \\
\hline Incident episodes of malaria & 38 & 12 & 20 & 6 \\
\hline Incidence of malaria per person years & 0.040 & 0.048 & 0.050 & 0.020 \\
\hline Prevalence of microscopic parasitemia* & 244/12,696 (1.9\%) & $40 / 3,352(1.2 \%)$ & $173 / 5,307$ (3.3\%) & $31 / 4,037(0.8 \%)$ \\
\hline $\begin{array}{l}\text { Geometric mean parasite density } / \mu \mathrm{L} \\
(95 \% \mathrm{Cl})\end{array}$ & $200(157-256)$ & $540(262-1,110)$ & $165(127-215)$ & $165(77-356)$ \\
\hline $\begin{array}{l}\text { Prevalence of microscopic or } \\
\text { submicroscopic parasitemia* }\end{array}$ & $1,314 / 12,695(10.4 \%)$ & $134 / 3,352(4.0 \%)$ & $743 / 5,306(14.0 \%)$ & $437 / 4,037(10.8 \%)$ \\
\hline $\begin{array}{l}\text { Geometric mean parasite density/ } \mu \mathrm{L} \\
(95 \% \mathrm{Cl})\end{array}$ & $3(2-3)$ & $6(3-13)$ & $5(4-6)$ & $1(1-1)$ \\
\hline $\begin{array}{l}\text { Prevalence of microscopic } \\
\text { gametocytemia* }\end{array}$ & $50 / 12,696(0.4 \%)$ & $15 / 3,352(0.5 \%)$ & $30 / 5,307(0.6 \%)$ & $5 / 4,037(0.1 \%)$ \\
\hline Prevalence of anemia $(\mathrm{Hb}<11 \mathrm{gm} / \mathrm{dL}) \dagger$ & $146 / 4,334(3.4 \%)$ & $101 / 1,147(8.8 \%)$ & $36 / 1813(2.0 \%)$ & $9 / 1,374(0.7 \%)$ \\
\hline
\end{tabular}



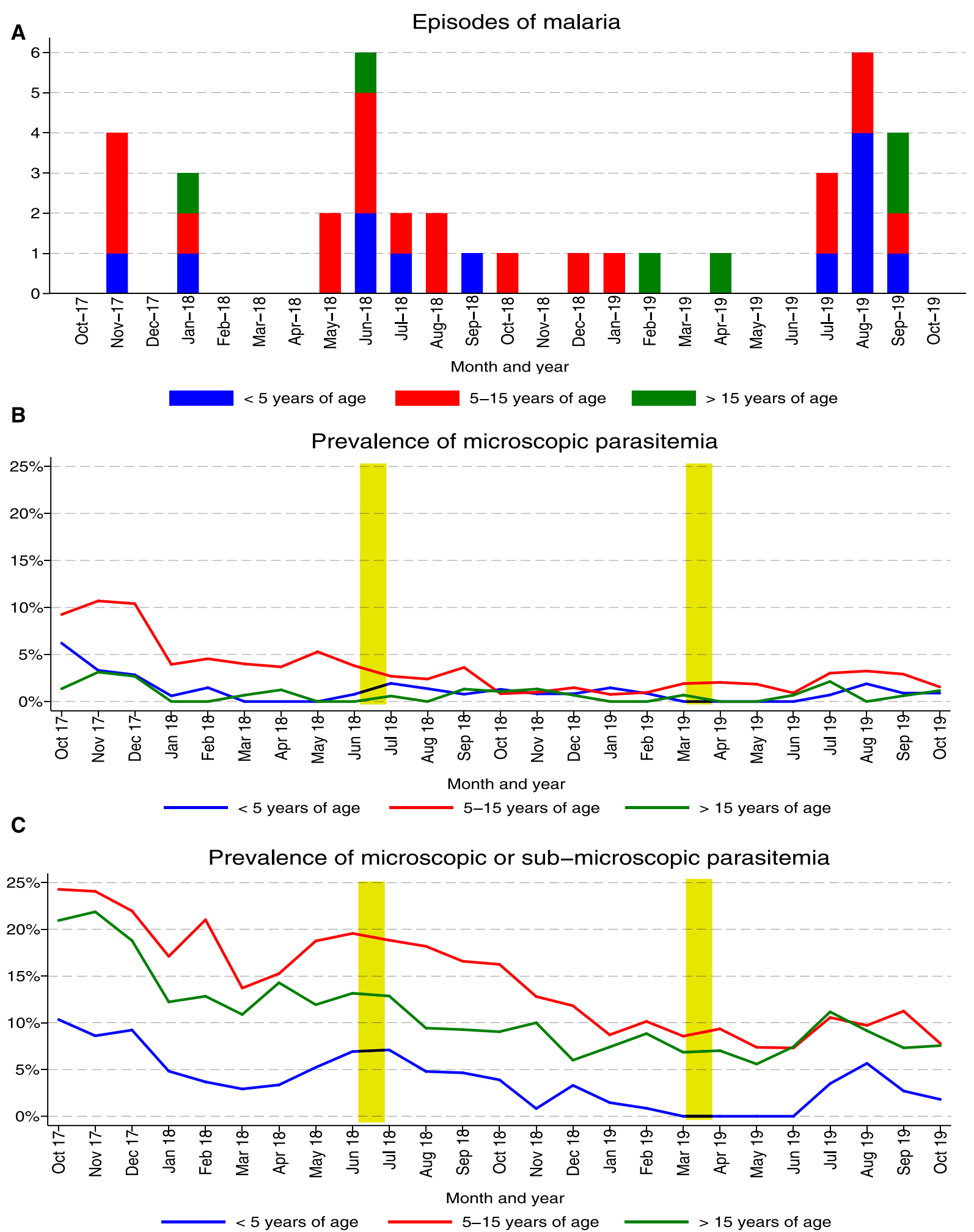

FIGURE 4. Temporal changes in the incidence of malaria and prevalence of parasitemia from the current cohort study (October 2017-October 2019). (A) Monthly episodes of symptomatic malaria stratified by age-group. (B) Monthly trends of microscopic parasitemia stratified by age-group. An interactive plot is available at $\mathrm{https}$ ://clinepidb.org/ce/app/workspace/strategies/import/7eeab4ea9951b3cd. (C) Monthly trends of microscopic or submicroscopic parasitemia stratified by age-group. (Yellow bars signify rounds of indoor residual spraying with pirimiphos-methyl.) This figure appears in color at www.ajtmh.org.

interesting observation after the implementation of IRS was the loss of a peak in transmission intensity following the October-November rainy season, resulting in only a single annual peak after the longer rains in March-May. Following the implementation of IRS, we also observed a dramatic shift in the predominate vector species from An. gambiae s.s. to An. arabiensis. This shift has also been reported previously from a study of human landing catches performed in the same region, 
which also documented an increase in the proportion of mosquitoes collected outdoors versus indoors following the implementation of IRS. ${ }^{19}$

Before the implementation of IRS, the incidence of malaria among children at our study site peaked at more than four episodes per year in children younger than 3 years, with a sharp decline to just more than one episode per year in children aged 3-10 years, as previously described. ${ }^{4}$ However, despite the high burden of malaria before IRS, less than $0.2 \%$ of episodes met the criteria for severe malaria, and there were no deaths due to malaria, which we attributed to access to prompt and effective treatment in our cohorts. Following 5 years of sustained IRS, there was a dramatic decline in the incidence of malaria, with a modest shift in the burden toward older children. In high endemic settings, repeated exposure to malaria parasites leads to the development of naturally acquired immunity, which provides protection against clinical disease in older age-groups. ${ }^{20}$ However, as transmission declines, it is expected that acquisition of immunity is lost or delayed, consistent with a shift of the burden of malaria to older children, as we observed following IRS. ${ }^{21}$ Although we only observed 38 cases of malaria in the $4-5$ years after IRS was implemented, it is comforting that there were no cases of severe malaria or deaths due to malaria. Thus, with prompt access to treatment, outcomes of malaria episodes remained excellent despite reduced parasite exposure and presumed delays in the acquisition of immunity. In addition, the prevalence of anemia was very low after sustained IRS, with moderate to severe anemia in only $0.3 \%$ in children younger than 5 years compared with findings from national MIS, in which the prevalence of moderate to severe anemia in this same agegroup was $10 \%$ in 2009 and $5 \%$ in $2014 .^{2,3}$ Indeed, we have previously reported significant increases in mean hemoglobin levels among children in our cohort studies following the implementation of IRS. ${ }^{22}$

As observed with measures of transmission and disease, there was a marked reduction in the prevalence of parasitemia following sustained IRS. After 5 years of IRS, the overall parasite prevalence by microscopy had fallen to $<2 \%$. However, when using a highly sensitive qPCR assay, most of the infections were submicroscopic such that the prevalence of combined microscopic and submicroscopic parasitemia was more than $10 \%$. In addition, the proportion of infections that were submicroscopic increased with age such that older children and adults were the primary foci of infection. The persistence of these largely asymptomatic, low-level infections in older children and adults likely has significant implications for malaria elimination in Uganda and other historically high-burden African countries. Achieving malaria elimination requires removal of the human reservoir of infection, including asymptomatic and submicroscopic infections, which are known to be transmissible to mosquitoes. ${ }^{23,24}$ These findings suggest that a combination of case management with ACTs, universal LLIN distribution, and IRS in a limited geographical setting may not be sufficient for the elimination of malaria in previously high transmission settings where An. arabiensis is present, and rather that combining vector control interventions with other interventions that target the human reservoir, such as regular chemoprevention, done at a large spatial scale may be needed to achieve elimination. ${ }^{25}$ The persistence of the human reservoir after 5 years of sustained IRS also raises concerns about IRS exit strategies. Indeed, withdrawal of IRS in a historically high-burden area of Northern Uganda was associated with a rapid resurgence of malaria burden to preIRS levels. ${ }^{26}$

This study had several limitations. First, the observational study design and lack of a contemporary control group limit our ability to make causal inferences about the impact of IRS on our measures of malaria transmission, disease, and infection. However, given continued implementation of LLINs and case management with ACTs, the magnitude of the changes observed, and their temporal relationship to IRS, it is highly likely that IRS was the major contributor to the observed declines. Second, different methods were used to detect submicroscopic parasitemia in our two cohort studies. Because the qPCR assay used in the second cohort study was more sensitive than the LAMP assay used in the first study, we likely underestimated the decline in the prevalence of combined microscopic and submicroscopic parasitemia over time. Third, entomological measures of transmission intensity, including the HBR and EIR, were estimated using CDC light traps and not standardized collection methods such as human landing catches. Therefore, caution should be taken when interpreting these estimates. Finally, our study cannot be considered representative of rural African settings, as participants in our cohort studies received higher quality medical care in a more timely manner than is typically available. However, over the course of our two cohort studies, the procedures we used to manage patients did not change.

We have demonstrated that, in a highly endemic area of Uganda, a combination of available malaria control interventions including case management with ACTs, universal LLIN distribution, and sustained IRS was associated with a dramatic reduction in the burden of malaria. The vision of the Uganda National Malaria Control Program is to have a malariafree Uganda by accelerating the nationwide scale-up of evidencebased malaria reduction interventions by the government, its development partners, the private sector, and all stakeholders. ${ }^{27}$ Uganda has already implemented a national policy of universal access to ACTs for case management and LLIN distribution repeated every 3-4 years. However, IRS is currently only being implemented in 14 of 135 districts because of limited resources. For Uganda to accelerate gains and achieve the success seen in Tororo district, additional resources to scale-up IRS to other high-burden areas will be of great value. In addition, even in areas where IRS has been implemented, there is a need to sustain this intervention, given the reservoir of largely asymptomatic infections that persisted after 5 years of IRS in Tororo and the risk of reintroduction from neighboring areas that have not achieved the same level of control.

Received April 6, 2020. Accepted for publication June 8, 2020.

Published online July 20, 2020.

Acknowledgments: We thank the study team and the Infectious Diseases Research Collaboration for administrative and technical support. We are grateful to the study participants who participated in this study and their families.

Financial support: Funding was provided by the National Institutes of Health as part of the International Centers of Excellence in Malaria Research (ICMER) program (U19AI089674). JIN is supported by the Fogarty International Center (Emerging Global Leader Award grant number K43TW010365). E. A. is supported by the Fogarty International Center of the National Institutes of Health under Award 
Number D43TW010526. The ClinEpiDB platform is supported by award OPP1169785 from the Bill \& Melinda Gates Foundation.

Disclosure: The funders had no role in the study design, data collection and analysis, decision to publish, or preparation of the manuscript.

Authors' addresses: Joaniter I. Nankabirwa, Emmanuel Arinaitwe, John Rek, Maxwell Kilama, Timothy Kizza, and Moses R. Kamya, Infectious Diseases Research Collaboration, Kampala, Uganda, E-mails: jnankabirwa@yahoo.co.uk, earinaitwe@idrc-uganda.org, jrek@ idrc-uganda.org, mkilama@idrc-uganda.org, tkizza@idrc-uganda. org, and mkamya@idrc-uganda.org. Sarah G. Staedke and Chris Drakeley, London School of Hygiene and Tropical Medicine, London, United Kingdom, E-mails: sarah.staedke@lshtm.ac.uk and chris. drakeley@Ishtm.ac.uk. Phillip J. Rosenthal, Isabel RodriguezBarraquer, Jessica Briggs, Bryan Greenhouse, and Grant Dorsey, University of California San Francisco, San Francisco, CA, E-mails: philip.rosenthal@ucsf.edu, isabel.rodriguez@ucsf.edu, jessica. briggs@ucsf.edu, bryan.greenhouse@ucsf.edu, and grant.dorsey@ ucsf.edu. Teun Bousema, Radboud University Nijmegen Medical Centre, Nijmegen, The Netherlands, E-mail: teun.bousema@ radboudumc.nl. David S. Roos and Sheena S. Tomko, University of Pennsylvania, Philadelphia, PA, E-mails: droos@sas.upenn.edu and stomko@sas.upenn.edu. David L. Smit, University of Washington, Seattle, WA, E-mail: smitdave@gmail.com.

\section{REFERENCES}

1. WHO, 2019. World Malaria Report. Geneva, Switzerland: World Health Organization. Available at: https://www.who.int/ publications-detail/world-malaria-report-2019. Accessed February 14, 2020.

2. Ministry of Health Uganda, 2009. Malaria Indicator Survey (MIS) 2009. Available at: https://dhsprogram.com/pubs/pdf/MIS6/ MIS6.pdf. Accessed February 26, 2020.

3. Health UMo, 2015. The Uganda Malaria Indicator Survey 2014-2015. Available at: https://dhsprogram.com/pubs/pdf/ mis21/mis21.pdf. Accessed March 15, 2020.

4. Kamya MR et al., 2015. Malaria transmission, infection, and disease at three sites with varied transmission intensity in Uganda: implications for malaria control. Am J Trop Med Hyg 92: 903-912.

5. Katureebe $A$ et al., 2016. Measures of malaria burden after longlasting insecticidal net distribution and indoor residual spraying at three sites in Uganda: a prospective observational study. PLoS Med 13: e1002167.

6. Nankabirwa Jl et al., 2019. Persistent parasitemia despite dramatic reduction in malaria incidence after 3 rounds of indoor residual spraying in Tororo, Uganda. $J$ Infect Dis 219: 1104-1111.

7. Zinszer $\mathrm{K}$ et al., 2020. The impact of multiple rounds of indoor residual spraying on malaria incidence and hemoglobin levels in a high-transmission setting. $J$ Infect Dis 221: 304-312.

8. Ministry of Health Uganda, 2016. Uganda Clinical Guidelines 2016. Available at: http://www.health.go.ug/content/ugandaclinical-guidelines-2016. Accessed February 12, 2020.

9. Gillies MT, Coetzee M, 1987. A Supplement to the Anophelinae of Africa South of the Sahara. Johannesburg, South Africa: South African Institute for Medical Research.

10. Scott JA, Brogdon WG, Collins FH, 1993. Identification of single specimens of the Anopheles gambiae complex by the polymerase chain reaction. Am J Trop Med Hyg 49: 520-529.

11. Wirtz RA, Duncan JF, Njelesani EK, Schneider I, Brown AE, Oster CN, Were JB, Webster HK, 1989. ELISA method for detecting
Plasmodium falciparum circumsporozoite antibody. Bull World Health Organ 67: 535-542.

12. Hofmann N, Mwingira F, Shekalaghe S, Robinson LJ, Mueller I, Felger I, 2015. Ultra-sensitive detection of Plasmodium falciparum by amplification of multi-copy subtelomeric targets. PLoS Med 12: e1001788.

13. Snounou G, Viriyakosol S, Zhu XP, Jarra W, Pinheiro L, do Rosario VE, Thaithong S, Brown KN, 1993. High sensitivity of detection of human malaria parasites by the use of nested polymerase chain reaction. Mol Biochem Parasitol 61: 315-320.

14. Kilama $M$ et al., 2014. Estimating the annual entomological inoculation rate for Plasmodium falciparum transmitted by Anopheles gambiae s.l. using three sampling methods in three sites in Uganda. Malar J 13: 111.

15. Administration, NAaS, 2011. TRMM and Other Data Precipitation Product. Washington, DC: National Aeronautics and Space Administration. Available at: https://disc.gsfc.nasa.gov/ mirador-guide. Accessed February 26, 2020.

16. Ruhamyankaka E et al., 2019. ClinEpiDB: an open-access clinical epidemiology database resource encouraging online exploration of complex studies. Gates Open Res 3: 1661.

17. WHO, 2015. An Operational for Manual Indoor Residual Spraying (IRS) for Malaria Transmission Control and Elimination, 2nd edition. Geneva, Switzerland: World Health Organization. Available at: https://apps.who.int/iris/bitstream/ handle/10665/177242/9789241508940_eng.pdf;jsessionid= 6335340CB2DAF21D00DAAA6A06777EAC? sequence $=1$. Accessed March 5, 2020.

18. Abiodun GJ, Maharaj R, Witbooi P, Okosun KO, 2016. Modelling the influence of temperature and rainfall on the population dynamics of Anopheles arabiensis. Malar J 15: 364.

19. Musiime AK et al., 2019. Impact of vector control interventions on malaria transmission intensity, outdoor vector biting rates and Anopheles mosquito species composition in Tororo, Uganda. Malar J 18: 445.

20. Yazdani SS, Mukherjee P, Chauhan VS, Chitnis CE, 2006. Immune responses to asexual blood-stages of malaria parasites. Curr Mol Med 6: 187-203.

21. Fowkes FJI, Boeuf P, Beeson JG, 2016. Immunity to malaria in an era of declining malaria transmission. Parasitology 143: 139-153.

22. Zinszer $\mathrm{K}$ et al., 2020. The impact of multiple rounds of indoor residual spraying on malaria incidence and hemoglobin levels in a high-transmission setting. $J$ Infect Dis 221: 304-312.

23. Lin JT, Saunders DL, Meshnick SR, 2014. The role of submicroscopic parasitemia in malaria transmission: what is the evidence? Trends Parasitol 30: 183-190.

24. Schneider P, Bousema JT, Gouagna LC, Otieno S, van de VegteBolmer M, Omar SA, Sauerwein RW, 2007. Submicroscopic Plasmodium falciparum gametocyte densities frequently result in mosquito infection. Am J Trop Med Hyg 76: 470-474.

25. Walker PGT, Griffin JT, Ferguson NM, Ghani AC, 2016. Estimating the most efficient allocation of interventions to achieve reductions in Plasmodium falciparum malaria burden and transmission in Africa: a modelling study. Lancet Glob Health 4: e474-e484.

26. Raouf $S$ et al., 2017. Resurgence of malaria following discontinuation of indoor residual spraying of insecticide in an area of Uganda with previously high-transmission intensity. Clin Infect Dis 65: 453-460.

27. Ministry of Health Uganda, 2014. The Uganda Malaria Reduction Strategic Plan 2014-2020. Available at: https://health.go.ug/ content/uganda-malaria-reduction-strategic-plan-2014-2020. Accessed March 18, 2020. 